Revue française de la traduction

\title{
Noms humoristiques de personnages et traditions traductives nationales
}

\section{Dominique Faria}

\section{(2) OpenEdition}

\section{Journals}

\section{Édition électronique}

URL : http://journals.openedition.org/traduire/702

DOI : $10.4000 /$ traduire.702

ISSN : 2272-9992

\section{Éditeur}

Société française des traducteurs

\section{Édition imprimée}

Date de publication : 15 juin 2015

Pagination : 99-105

ISSN : 0395-773X

\section{Référence électronique}

Dominique Faria, « Noms humoristiques de personnages et traditions traductives nationales », Traduire [En ligne], 232 | 2015, mis en ligne le 15 juin 2017, consulté le 21 avril 2019. URL : http:// journals.openedition.org/traduire/702 ; DOI : 10.4000/traduire.702 


\section{Noms humoristiques de personnages et traditions traductives nationales}

\section{Dominique Faria}

Les études sur la traduction ont tendance à comparer texte de départ et texte d'arrivée pour en signaler les disparités et les attribuer soit aux différences entre les deux langues et/ou cultures, soit aux choix individuels des traducteurs. Dans cet article on se propose de réfléchir sur un troisième facteur, extralinguistique : la tradition de traduction nationale.

La Routledge Encyclopedia of Translation Studies (deuxième édition), consacre une section conséquente(1) à la présentation, par pays, de l'histoire de la traduction, de la formation des traducteurs, des grands théoriciens ou des traducteurs célèbres. Ce contexte dans lequel travaille le traducteur, bien que difficile à cerner, n'en joue pas moins un rôle essentiel dans sa conception de la traduction.

Pour aborder cette thématique, on s'interrogera sur la traduction de l'humour linguistique. Selon Raphaelson-West $(1989,31)$, des trois types d'humour - linguistique, culturel et universel - le premier s'avère le plus difficile à traduire, étant donné les spécificités et les limitations de chaque langue nationale. Nous suggérons ici que la principale difficulté n'est précisément pas à rechercher dans les différences entre les langues.

Nous nous arrêterons sur la traduction d'un cas spécifique d'humour linguistique, celui que produisent les noms de personnages, que nous étudierons à partir d'un corpus composé de deux romans anglais publiés à la fin du xxe siècle et traduits en français, portugais européen, portugais du Brésil et espagnol. Le choix de ces langues cible, issues du latin et possédant par conséquent des ressources linguistiques similaires, permettra de mieux signaler les différentes approches traductives selon les pays.

Le processus de traduction résultant d'une suite de prises de décision, sans pouvoir aboutir à une solution optimale (Levý, 2000), la première d'entre elles qui s'impose, dans le cas des noms de personnages, est traduire ou ne pas traduire. II existe une contradiction entre le

(1) « Part II: History and Traditions », p. 313-550. 
besoin de créer un texte à effet humoristique dans la langue d'arrivée et l'usage généralement accepté qui consiste à ne pas traduire les noms propres(2).

La traduction des noms propres à effet humoristique permet de préserver la composante humoristique et ludique du texte. Les noms propres étant souvent utilisés par l'auteur pour caractériser les personnages, le lecteur de la traduction n'aura accès à ces informations que si les noms sont traduits ou s'il connait (bien) la langue de départ. En revanche, ne pas traduire a l'avantage de garder le nom choisi par l'auteur, avec ses connotations nationales et ses sonorités, et de faciliter la communication sur le livre avec des lecteurs d'autres nationalités. Cela permet aussi de souligner l'origine étrangère du texte et, par conséquent, le fait qu'il s'agit d'une traduction, provoquant le fameux effet d'étrangeté, ou d'étrangèreté (Berman, 1984, Venuti, 1995).

Traduire l'humour linguistique exige du traducteur qu'il soit, tout comme l'auteur, un habile humoriste et qu'il fasse preuve de créativité. Or, pour cela, il faut que la culture d'arrivée accepte ce genre d'intervention du traducteur sur le texte. On retrouve ici le sujet le plus débattu de la longue histoire des études sur la traduction - depuis Cicéron et Saint Jérôme et l'éternel choix entre fidélité (à l'auteur, au texte) et liberté. La décision de traduire les noms des personnages correspond à une conception créative de la traduction. Autrement dit, le traducteur aura tendance à traduire les noms de personnages lorsque la tradition de traduction nationale dans/pour laquelle il travaille l'y autorise ou l'encourage à le faire.

\section{La traduction des noms de personnages à effet humoristique : quelques exemples}

Les premiers exemples sur lesquels nous nous arrêterons portent sur le roman de l'écrivain britannique Terry Pratchett, Wyrd Sisters, sixième volume de la série Discworld. Ce roman, comme les autres de la série, appartient au genre fantasy. Publié en Angleterre en 1988, il a été traduit en France en 1995, au Portugal en 1991, au Brésil en 2003 et en Espagne en 1992(3). L'humour linguistique, caractéristique de l'écriture de l'auteur, y est omniprésent. II est visible dans les noms des sorcières:

(Anglais) Nanny Ogg, Granny Weatherwax, Magrat Garlick, Goodie Whemper

(Français) Nounou Ogg, Mémé Ciredutemps, Magrat Goussedail, Bobonne Pleurniche

(Portugais - Portugal) Nanny Ogg, Granny Weatherwax, Magrat Garlick, Goodie Whemper

(2) D'ailleurs, comme le souligne Lia Wyler $(2003,15)$ à propos de la traduction de la littérature jeunesse au Brésil, les contrats imposent souvent aux éditeurs de garder les noms propres dans la langue de départ, ce qui prive les lecteurs de l'humour qu'ils recèlent.

(3) Pour les éditions utilisées dans cette étude, voir la bibliographie. 
(Portugais - Brésil) Tia Ogg, Vovó Cera do Tempo, Margrete Alho, Dona Lamória (Espagnol) Tata Ogg, Yaya Ceravieja, Magrat Ajostiernos, Abuela Whemper

Ces noms sont formés de deux parties. La première partie - Nanny (nounou), Granny (mémé) et Goodie (qui renvoie à bon), mais on notera que Magrat, une jeune sorcière, constitue une exception - joue, en l'inversant, sur la réputation maléfique des sorcières. La seconde évoque le caractère du personnage : Weatherwax (cire du temps), Garlick (ail) et Whemper (composé à partir de "whimper ", gémissement). Nanny Ogg, par exemple, a un grand nombre d'enfants et de petits-enfants dont elle doit s'occuper et Granny Weatherwax est l'une des personnes les plus âgées du pays, une autorité, respectée de tous.

En examinant les solutions proposées par les traducteurs, on constate que la décision du traducteur portugais diffère manifestement de celle de ses confrères. En effet, le nom de ces personnages a été traduit dans les autres langues, reproduisant les connotations associées aux noms et aux personnages dans la langue de départ(4). Les traducteurs ont donc maintenu l'aspect humoristique et ludique du texte de départ, afin de produire sur le lecteur de la traduction un effet similaire à celui de la version originale sur le lecteur anglais.

Le traducteur portugais a, quant à lui, produit un texte moins humoristique. On constate par ailleurs que, à l'inverse des traducteurs des autres nationalités, il a souvent recours aux notes de traduction, généralement pour indiquer l'impossibilité de traduire tel ou tel jeu de mots. Une de ces notes porte sur un jeu de mots filé. Le traducteur y avoue avoir produit un passage qui n'a pas de sens et explique sa décision de ne pas adapter le texte : "Aqui trata-se, porém, de T. Pratchett... " [II s'agit, cependant, de T. Pratchett]. La raison de la non-traduction n'est donc point l'inexistence de ressources linguistiques correspondantes en portugais, mais plutôt le respect de l'auteur, qui empêche le traducteur de modifier le texte. La traduction est perçue ici comme un texte-second par rapport à l'original.

Arrêtons-nous maintenant brièvement sur quelques noms des personnages tirés des fameux romans de la série Harry Potter, plus précisément de Harry Potter and the Philosopher's Stone. Comme dans le cas précédent, les jeux de langue, caractéristiques de l'écriture de J.K. Rowling, rendent son texte humoristique et ludique. Ainsi, les professeurs et les auteurs des livres étudiés à l'école de sorcellerie de Hogwarts ont des noms qui évoquent leur spécialité : le professeur de botanique s'appelle Sprout (pousse, mais aussi chou de Bruxelles), l'auteur d'un important livre de botanique s'appelle Spore (spore), celui d'un livre sur la transfiguration a pour nom Switch (modifier). Ces noms n'ont pas été traduits dans les langues avec lesquelles nous travaillons, à une exception : dans la version française, ces personnages sont appelés respectivement Chourave, Augirole et Changé.

(4) Les menues différences entre les solutions trouvées peuvent être attribuées aux interprétations et préférences du traducteur ou aux connotations des mots dans chaque langue. 
Les noms des deux chiens du géant Hagrid servent aussi à illustrer notre propos : l'un - Fluffy (pelucheux) - est un féroce chien à trois têtes, et l'autre - Fang (croc) - est très gentil et peureux. Ces noms n'ont pas non plus été traduits au Portugal et en Espagne. En France, ils sont devenus "Touffu " et "Crockdur " et au Brésil "Fofo " (pelucheux) et "Canino " (qui signifie "féroce " et/ou " dent "), des traductions qui reproduisent les connotations de la version anglaise.

Sans la traduction, il devient difficile pour les jeunes lecteurs d'avoir accès à ces jeux de mots. Le cas de la série Harry Potter est assez particulier, car un autre facteur extralinguistique a influencé les choix de traduction: après l'achat des droits cinématographiques par Warner Brothers, il a été demandé aux maisons d'édition de ne pas traduire les noms des personnages, ce qui explique qu'ils aient été conservés en Espagne et (partiellement) au Brésil. Cette restriction ne fait que souligner la liberté dont ont joui les traducteurs français, qui constituent, dans le cas présent, une exception en Europe.

\section{Les traditions traductives nationales}

Bien que les cas présentés ne soient pas suffisamment nombreux pour en tirer des conclusions définitives, ils nous semblent représentatifs des tendances de la traduction à la fin du xxe siècle et au début du Xxı siècle dans ces quatre pays. Si on devait classer les traducteurs en fonction de leur degré d'intervention sur le texte et de leur degré de créativité, les Français occuperaient un pôle, les Portugais le pôle opposé, les Espagnols et les Brésiliens se trouvant en position intermédiaire.

Ainsi, les traducteurs français ont tendance à traduire les noms de personnages, à être plus créatifs, à modifier le sens du texte au profit d'autres qualités jugées importantes. Un bon exemple en est la traduction de "Fang " par "Crockdur ", sur la base du mot français " croc " ou l'emploi de "Pleurniche " pour remplacer "Whemper "(5). Les traducteurs espagnols et brésiliens ne sont pas aussi créatifs, même s'ils ont tendance à traduire les noms des personnages et à (re)créer l'humour linguistique. Les traducteurs portugais, en revanche, choisissent plus souvent de maintenir le nom des personnages dans la langue de départ.

Or, il nous semble que ces tendances sont en partie déterminées par la tradition traductive de chaque pays(6). Si on compare la tradition française et la tradition portugaise, on constate

(5) D'ailleurs, le traducteur français de Terry Pratchett, Patrick Couton, a reçu le Grand Prix de I'Imaginaire en 1998 et le Prix Imaginales - Prix Spécial du Jury, en 2002, pour ses traductions des volumes du Discworld.

(6) Évidemment, d'autres facteurs contribuent à déterminer les choix des traducteurs. On peut penser, pour les traducteurs français, au rôle joué par la longue tradition littéraire française d'approche ludique de la langue (dont témoigne par exemple le mouvement de l'OuLiPo), qui rend traducteurs et lecteurs plus sensibles à l'humour linguistique. 
qu'en France un débat public autour de la traduction a lieu depuis plusieurs siècles, qu'un grand nombre de théoriciens incontournables étaient français, que la formation de traducteurs est très répandue et valorisée. Quant au Portugal, il suffit de dire qu'il ne fait pas partie des 31 pays dont la Routledge Encyclopedia of Translation Studies étudie les traditions de traduction. Notons en outre que la recherche sur la traduction y est assez récente, la formation des traducteurs également(7). Dans une étude datée de 2006, Jorge Pinho a entrepris une analyse systématique des discours des traducteurs portugais du xxe siècle, s'étant aperçu que leur plus grand souci était la fidélité à l'auteur. Les traductions portugaises effectuées au cours du xxe siècle suivent donc généralement une stratégie qui rend compte de l'altérité du textesource, conservant un grand nombre de mots étrangers et de structures syntaxiques qui diffèrent de celles du portugais(8).

Pour ce qui est du Brésil, le fameux mouvement de l'anthropophagie, mené par Haroldo de Campos, y a joué un rôle crucial pour rendre aux traducteurs brésiliens la liberté d'intervenir dans le texte. En Espagne, les traducteurs bénéficient aussi d'une marge de liberté bien plus grande qu'au Portugal. Selon Miguel Vega $(2004,567)$, la tendance du xxe siècle est à la production de textes qui semblent avoir été écrits directement en espagnol. L'article de la Routledge Encyclopedia sur la traduction de tradition espagnole mentionne d'ailleurs la propension à hispaniser les noms propres (Antony Pym, 2001, 559), laquelle perdure tout au long du xxe siècle.

Sans trop vouloir nous étendre - notre propos n'étant pas de saisir chaque tradition nationale, mais plutôt d'en signaler la place dans l'équation complexe de la traduction - soulignons que ce sont là des tendances et des attentes que le traducteur doit prendre en considération.

\section{En conclusion}

La traduction n'est donc point une question strictement linguistique et le traducteur n'est pas toujours totalement libre de ses décisions. Outre les difficultés linguistiques provenant des différences entre les langues et même de la capacité du traducteur à identifier et à (re)créer l'humour linguistique, des facteurs extralinguistiques comme les traditions traductives nationales déterminent largement le choix des traducteurs. Seul un traducteur travaillant dans un environnement où son intervention sur le texte, sa créativité et son autorité sont reconnues cherchera à reproduire l'humour du texte de départ, lorsque celui-ci implique d'y apporter des modifications importantes au niveau du sens.

dominiquefaria@uac.pt

(7) À ce propos, voir Faria (2013).

(8) Parmi les quatre pays mentionnés dans cet article, c'est aussi le seul à proposer des programmes de télévision qui ne soient pas systématiquement doublés. En général, seuls les programmes pour enfants sont doublés au Portugal. 


\section{Bibliographie}

\section{Corpus}

PRATCHETT Terry, 1988, Wyrd Sisters, London, Corgi.

PRATCHETT Terry, 1995, Trois sœurcières, traduction de Patrick Couton, Paris, L'Atalante.

PRATCHETT Terry, 2003, Estranhas Irmãs, traduction de Roberto DeNice, São Paulo, Conrad Editora.

PRATCHETT Terry, 2005 (1re éd. 1991), As Três Bruxas, traduction de Mário Dias Correia, Lisboa, Temas e Debates.

PRATCHETT Terry, 2010 (1re éd. 1992), Brujerías, traduction de Cristina Macía, Barcelona, Debolsillo.

ROWLING J.-K., 1997, Harry Potter and the Philosopher's Stone, London, Bloomsbury.

ROWLING J.-K., 1998, Harry Potter à l'école des sorciers, traduit par Jean-François Ménard, Paris, Gallimard.

ROWLING J.-K., 1999a, Harry Potter e a pedra filosofal, traduit par Isabel Fraga, Lisboa, Presença.

ROWLING J.-K., 1999b, Harry Potter y la piedra filosofal, traduit par Alicia Dellepiane Rawson, Barcelona, Salamandra.

ROWLING J.-K., 2000, Harry Potter e a pedra filosofal, traduit par Lia Whyler, Rio de Janeiro, Editora Rocco.

\section{Études}

BAKER Mona (éd.), 2001, Routledge Encyclopedia of Translation Studies, London, New York, Routledge.

BERMAN Antoine, 1984, L'épreuve de l'étranger. Culture et traduction dans l'Allemagne romantique, Paris, Gallimard.

FARIA Dominique, 2013, "La revue Colóquio Letras et la mise en valeur de la traduction au Portugal ", in Constantinescu Muguras, Haisan Daniela (coord.), Atelier de traduction, Dossier Critique des Traductions II, Romenia, Editura Universitatii din Suceava, p. 95-108.

LEVÝ Jiri, 2000, (1re éd. 1967) "Translation as a decision process", in VENUTI Lawrence, (ed.), The Translation Studies Reader, London, New York, Routledge, p. 148-159. 
PINHO Jorge Almeida, 2006, 0 escritor invisível. A tradução tal como é vista pelos tradutores portugueses, Matosinhos, Quidnovi.

PYM Anthony, 2001, "Spanish tradition", in Baker Mona (éd.), Routledge Encyclopedia of Translation Studies, London, New York, Routledge, p. 552-563.

RAPHAELSON-WEST Debra, 1989, "On the feasibility and strategies of translating humour", Meta, vol. 34, n 1, p. 128-141.

VEGA Miguel Ángel, 2004, "De la Guerra Civil al pasado inmediato", in LAFARGA Francisco, PEGENAUTE Luis (éd.), Historia de la traducción en España, Salamanca, Editorial Ambos Mundos, p. 527-578.

VENUTI Lawrence, 1995, The Translator's Invisibility, London, New York, Routledge.

WYLER Lia, 2003, Línguas, poetas e bacharéis - Uma crônica da tradução no Brasil, Rio de Janeiro, Rocco.

Dominique Faria est membre du Centro de Estudos Comparatistas de l'Université de Lisbonne et Maître de conférences à l'Université des Açores, au Portugal, où elle enseigne la littérature française et la théorie de la traduction. Elle est Docteur en littérature française, avec une thèse sur Jean Echenoz, Éric Chevillard et Christian Gailly, et ses domaines de recherche sont le roman français contemporain et la traduction, sujets qui lui ont valu de participer à des colloques et de publier différents articles et chapitres de livres. Elle est sous-directrice de la revue Carnets et membre du comité scientifique et du comité de lecture de Fert'îles (Université de Corse). 